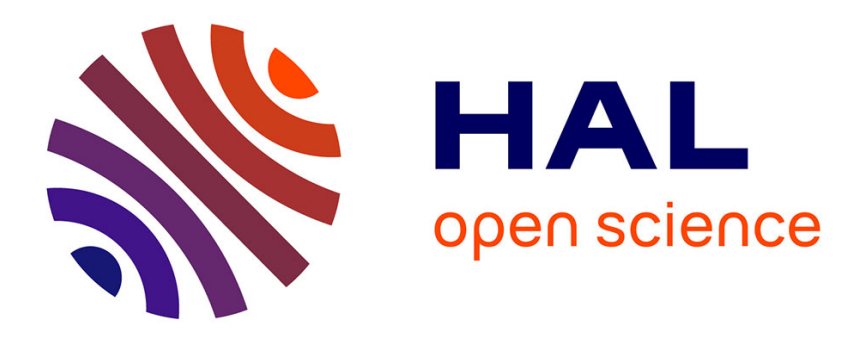

\title{
Dynamic substructuring in the medium-frequency range
}

\author{
Christian Soize, S. Mziou
}

\section{To cite this version:}

Christian Soize, S. Mziou. Dynamic substructuring in the medium-frequency range. 1st International ISMA Workshop on Noise and Vibration in Agricultural and Biological Engineering, Sep 2000, Leuven, Belgium. pp.Pages: 1185-1191. hal-00686291

\section{HAL Id: hal-00686291 https://hal.science/hal-00686291}

Submitted on 9 Apr 2012

HAL is a multi-disciplinary open access archive for the deposit and dissemination of scientific research documents, whether they are published or not. The documents may come from teaching and research institutions in France or abroad, or from public or private research centers.
L'archive ouverte pluridisciplinaire HAL, est destinée au dépôt et à la diffusion de documents scientifiques de niveau recherche, publiés ou non, émanant des établissements d'enseignement et de recherche français ou étrangers, des laboratoires publics ou privés. 


\title{
Dynamic Substructuring in the Medium-Frequency Range
}

\author{
C. Soize and S. Mziou \\ Structural Dynamics and Coupled Systems Department \\ ONERA, BP 72, 92322 Chatillon Cedex, France \\ e-mail : mziou@onera.fr \\ e-mail : soize@onera.fr
}

\begin{abstract}
There are several methods in dynamic substructuring for numerical simulation of complex structures in the lowfrequency range, that is to say in the modal range. For instance, the Craig-Bampton method is a very efficient and popular method in linear structural dynamics. Such a method is based on the use of the first structural modes of each substructure with fixed coupling interface. In the medium-frequency range, i.e. in the nonmodal range, and for complex structures, a large number of structural modes should be computed with finite element models having a very large number of degrees of freedom. Such an approach would not be efficient at all and generally, cannot be carried out. In this paper, we present a new approach in dynamic substructuring for numerical calculation of complex structures in the medium-frequency range. This approach is still based on the use of the Craig-Bampton decomposition of the admissible displacement field but the reduced matrix model of each substructure with fixed coupling interface, which is not constructed using the structural modes, is constructed using the first eigenfunctions of the mechanical energy operator of the substructure with fixed coupling interface related to the medium-frequency band. The method and a numerical example is presented.
\end{abstract}

\section{Introduction}

In the low-frequency range, the Craig-Bampton method [2] is very efficient to calculate the dynamical response of a complex structure modeled by finite element method. This method was initialy developped for discretized systems. The continuous version for a conservative structure can be found in [4], [5] and for a dissipative structure in [6], [7]. This method is based on the use of the structural modes of each substructure with fixed coupling interface allowing a reduced matrix model to be constructed. It is known that structural modes cannot be used to construct such a reduced matrix model in the mediumfrequency range for many reasons (see for instance [10], [7]). Recently, a method was proposed to construct reduced matrix model in the medium-frequency (MF) range [8]-[9]. In this paper, we present a new approach for dynamic substructuring in the MF range. This approach is similar to Craig-Bampton method, but the structural modes for each substructure with fixed coupling interface are replaced by the eigenfunctions associated with the highest eigenvalues of the mechanical energy operator related to the MF band for the substructure. In section 2, we present the dynamic substructuring construction in the MF range.
Section 3 deals with the construction of eigenvector basis used for the reduced matrix model of each substructure in the MF range. Finally, an example is presented in section 4 .

\section{Dynamic substructuring con- struction in the medium- frequency range.}

In this paper, the formulation is written in the frequency domain and is based on the use of a finite element model. In addition, it is assumed that the structure is subdivided into two substructures. Generalization to several ones is straightforward.

\subsection{Reduced matrix model for a sub- structure.}

We consider linear vibrations of a 3D viscoelastic structure around a static configuration considered as a natural state (without prestresses). The structure is fixed and occupies a bounded domain $\Omega$ of $\mathbb{R}^{3}$, with boundary $\partial \Omega=\Gamma \cup \Gamma_{0}$ where $\Gamma_{0}$ is the part of the boundary in which the displacement field is zero (Dirichlet conditions). The outward unit normal to 


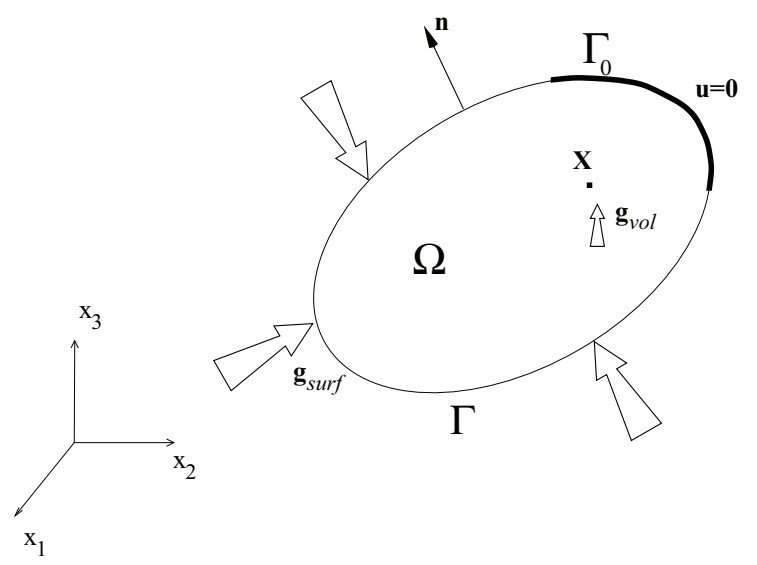

Figure 1: Geometrical configuration.

$\partial \Omega$ is denoted by $\mathbf{n}$ (see Figure 1 ). We introduce the narrow MF band defined by

$$
B=\left[\omega_{B}-\frac{\Delta \omega}{2}, \omega_{B}+\frac{\Delta \omega}{2}\right] \subset \mathbb{R}^{+},
$$

in which $\omega_{B}$ is the central frequency of the band and $\Delta \omega$ is the bandwidth such that

$$
\frac{\Delta \omega}{\omega_{B}} \ll 1 \quad, \quad \omega_{B}-\frac{\Delta \omega}{2}>0 .
$$

The interval $\widetilde{B}$ defined by

$$
\widetilde{B}=\left[-\omega_{B}-\frac{\Delta \omega}{2},-\omega_{B}+\frac{\Delta \omega}{2}\right],
$$

is associated with $B$. The structure is submitted to an external body force field $\left\{\mathbf{g}_{\text {vol }}(\mathbf{x}, \omega), \mathbf{x} \in \Omega\right\}$ and a surface force field $\left\{\mathbf{g}_{\text {surf }}(\mathbf{x}, \omega), \mathbf{x} \in \Gamma\right\}$, in which $\omega \in B \cup \widetilde{B}$. Structure $\Omega$ is decomposed into two substructures $\Omega_{1}$ and $\Omega_{2}$ whose coupling interface is $\Sigma$. The boundaries of $\Omega_{1}$ and $\Omega_{2}$ are written as $\partial \Omega_{1}=\Gamma_{1} \cup \Sigma$ and $\partial \Omega_{2}=\Gamma_{0} \cup \Gamma_{2} \cup \Sigma$ respectively (see Figure 2 ). We consider finite element meshes of $\Omega_{1}$ and $\Omega_{2}$ which are assumed to be compatible on coupling interface $\Sigma$. For $\omega$ in $B \cup \widetilde{B}$ and $r \in\{1,2\}$, we introduce the $\mathbb{C}^{n_{r}}$-valued vectors $\mathbf{U}^{r}(\omega), \mathbf{F}^{r}(\omega)$ and $\mathbf{F}_{\Sigma}^{r}(\omega)$ constitued of the $n_{r}$ DOFs, the discretized forces induced by external forces $\mathbf{g}_{o l}$ and $\mathbf{g}_{\text {surf, }}$ and the discretized internal coupling forces applied to coupling interface $\Sigma$, respectively. The matrix equation for substructure $\Omega_{r}$ is then written as

$$
\left[A^{r}(\omega)\right] \mathbf{U}^{r}(\omega)=\mathbf{F}^{r}(\omega)+\mathbf{F}_{\Sigma}^{r}(\omega) .
$$

in which symmetric $\left(n_{r} \times n_{r}\right)$ complex matrix $\left[A^{r}(\omega)\right]$ is the dynamical stiffness matrix of substructure $\Omega_{\text {r }}$ with a free coupling interface, which is defined by

$$
\left[A^{r}(\omega)\right]=-\omega^{2}\left[M^{r}\right]+i \omega\left[D^{r}(\omega)\right]+\left[K^{r}(\omega)\right],
$$

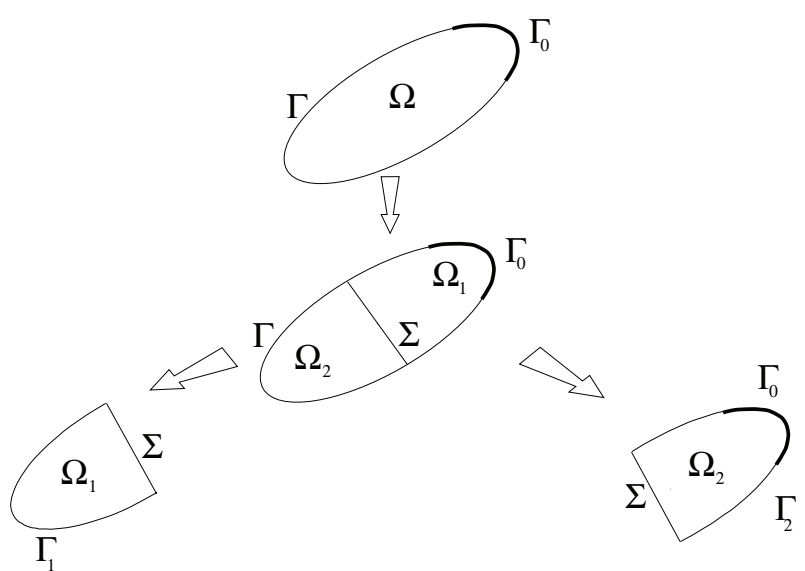

Figure 2: Structure decomposed into 2 substructures.

where $\left[M^{r}\right],\left[D^{r}(\omega)\right]$ and $\left[K^{r}(\omega)\right]$ are positive symmetric $\left(n_{r} \times n_{r}\right)$ real matrices. It should be noted that the damping and stiffness matrices depend on frequency $\omega$ because the material is viscoelastic. In addition, since $\omega=0$ does not belong to $B \cup \widetilde{B}$, for all $\omega \in B \cup \widetilde{B}$, matrix $\left[A^{1}(\omega)\right]$ of the free substructure $\Omega_{1}$ is invertible (matrix $\left[M^{1}\right]$ is positive definite and matrices $\left[D^{1}(\omega)\right],\left[K^{1}(\omega)\right]$ are only positive), while matrix $\left[A^{2}(\omega)\right]$ of the fixed substructure $\Omega_{2}$ is invertible for any real $\omega$ (matrices $\left[M^{2}\right],\left[D^{2}(\omega)\right],\left[K^{2}(\omega)\right]$ are positive definite). Vector $\mathbf{U}^{r}(\omega)$ is written as $\mathbf{U}^{r}(\omega)=\left(\mathbf{U}_{i}^{r}(\omega), \mathbf{U}_{j}^{r}(\omega)\right)$ in which $\mathbf{U}_{i}^{r}(\omega)$ is the $\mathbb{C}^{n_{r}-m}$-valued vector of the $n_{r}-m$ internal DOFs and $\mathbf{U}_{j}^{r}(\omega)$ is the $\mathbb{C}^{m}$-valued vector of the $m$ coupling DOFs. Consequently, matrix $\left[A^{r}(\omega)\right]$ and vector $\mathbf{F}^{r}(\omega)+\mathbf{F}_{\Sigma}^{r}(\omega)$ can be written as

$$
\begin{aligned}
{\left[A^{r}(\omega)\right] } & =\left[\begin{array}{cc}
{\left[A_{i i}^{r}(\omega)\right]} & {\left[A_{i j}^{r}(\omega)\right]} \\
{\left[A_{i j}^{r}(\omega)\right]^{T}} & {\left[A_{j j}^{r}(\omega)\right]}
\end{array}\right], \\
\mathbf{F}^{r}(\omega)+\mathbf{F}_{\Sigma}^{r}(\omega) & =\left[\begin{array}{c}
\mathbf{F}_{i}^{r}(\omega) \\
\mathbf{F}_{j}^{r}(\omega)+\mathbf{F}_{\Sigma, j}^{r}(\omega)
\end{array}\right],
\end{aligned}
$$

in which exponent $T$ denotes the transpose of matrices, and where $\mathbf{F}_{\Sigma}^{r}=\left(0, \mathbf{F}_{\Sigma, j}^{r}\right) \in \mathbb{C}^{n_{r}-m} \times \mathbb{C}^{m}$. The coupling conditions on interface $\Sigma$ are written as

$$
\begin{gathered}
\mathbf{U}_{j}^{1}(\omega)=\mathbf{U}_{j}^{2}(\omega)=\mathbf{U}_{j}(\omega), \\
\mathbf{F}_{\Sigma, j}^{1}(\omega)+\mathbf{F}_{\Sigma, j}^{2}(\omega)=0 .
\end{gathered}
$$

The Craig-Bampton method [2] introduced for finite element models is based on a fundamental mathematical property proved for the boundary value problems in Ref. [4], consisting in writing (see Figure 3) the admissible displacement vector space $\mathcal{C}_{r}$ for substructure $\Omega_{r}$ with free coupling interface $\Sigma$ as the direct sum of the vector space $\mathcal{C}_{r, \Sigma}$ of static liftings relative to coupling interface $\Sigma$ (so called the space of 

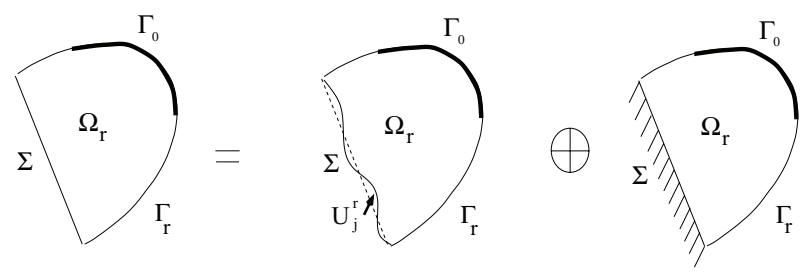

Figure 3: Substructuring principle.

static boundary functions) with the admissible displacement vector $\mathcal{C}_{r, 0}$ for substructure $\Omega_{r}$ with fixed coupling interface $\Sigma$,

$$
\mathcal{C}_{r}=\mathcal{C}_{r, \Sigma} \oplus \mathcal{C}_{r, 0}
$$

We then propose an approach for dynamic substructuring in the MF range which is based on the use of the fundamental property defined by Eq. (10) and on the construction of a reduced matrix model for substructure $\Omega_{r}$ with fixed interface $\Sigma$. This construction is obtained in substituting the structural modes of the associated conservative substructure by the eigenfunctions associated with the highest positive eigenvalues of the mechanical energy operator of this substructure, relative to MF band $B \cup \widetilde{B}$ (see [8]-[9]). It should be noted that structural modes can only be used in the LF range and cannot be used in the MF range [10], [7]. In addition, we propose to construct space $\mathcal{C}_{r, \Sigma}$ in considering the static liftings associated with the stiffness operator at the central frequency $\omega_{B}$ of band $B$. When applied to the finite element model, this theory leads us to write

$$
\mathbf{U}_{i}^{r}(\omega)=\left[\Phi_{i j}^{r}\right] \mathbf{U}_{j}^{r}(\omega)+\left[P^{r}\right] \mathbf{q}^{r}(\omega),
$$

in which $\left[\Phi_{i j}^{r}\right]$ is the $\left(n_{r}-m, m\right)$ real matrix defined by

$$
\left[\Phi_{i j}^{r}\right]=-\left[K_{i i}^{r}\left(\omega_{B}\right)\right]^{-1}\left[K_{i j}^{r}\left(\omega_{B}\right)\right] .
$$

Matrix $\left[P^{r}\right]$ is the $\left(n_{r}-m, N_{r}\right)$ real matrix whose columns are the eigenvectors associated with the $N_{r}$ highest eigenvalues of the matrix of the mechanical energy operator relative to band $B \cup \widetilde{B}$ and constructed by the finite element method. Vector $\boldsymbol{q}^{\prime}(\omega)$ is the $\mathbb{C}^{N_{r}}$-valued vector of the generalized coordinates. Consequently, physical DOFs can be written with respect to $\left\{\mathbf{q}^{r}(\omega), \mathbf{U}_{j}^{r}(\omega)\right\}$ as

$$
\left[\begin{array}{c}
\mathbf{U}_{i}^{r}(\omega) \\
\mathbf{U}_{j}^{r}(\omega)
\end{array}\right]=\left[\begin{array}{cc}
{\left[P^{r}\right]} & {\left[\Phi_{i j}^{r}\right.} \\
{[0]} & {\left[I_{m}\right]}
\end{array}\right]\left[\begin{array}{c}
\mathbf{q}^{r}(\omega) \\
\mathbf{U}_{j}^{r}(\omega)
\end{array}\right],
$$

in which $\left[I_{m}\right]$ is the $(m, m)$ unity matrix. The $\left(n_{r}, N_{r}+m\right)$ real matrix on the right-hand side of Eq. (13) being denoted by $\left[H^{r}\right]$, we deduce that the reduced matrix model associated with Eq. (4) is defined by the matrix $\left[\mathcal{A}^{r}(\omega)\right]$ and the vector $\mathcal{F}^{r}(\omega)$ such that

$$
\begin{aligned}
{\left[\mathcal{A}^{r}(\omega)\right] } & =\left[H^{r}\right]^{T}\left[A^{r}(\omega)\right]\left[H^{r}\right] \\
\mathcal{F}^{r}(\omega) & =\left[H^{r}\right]^{T}\left(\mathbf{F}^{r}(\omega)+\mathbf{F}_{\Sigma}^{r}(\omega)\right),
\end{aligned}
$$

which is rewritten with respect to $\left\{\mathbf{q}^{r}(\omega), \mathbf{U}_{j}^{r}(\omega)\right\}$ as

$$
\begin{aligned}
{\left[\mathcal{A}^{r}(\omega)\right] } & =\left[\begin{array}{cc}
{\left[\mathcal{A}_{i i}^{r}(\omega)\right]} & {\left[\mathcal{A}_{i j}^{r}(\omega)\right]} \\
{\left[\mathcal{A}_{i j}^{r}(\omega)\right]^{T}} & {\left[\mathcal{A}_{j j}^{r}(\omega)\right]}
\end{array}\right], \\
\mathcal{F}^{r}(\omega) & =\left[\begin{array}{c}
\mathcal{F}_{i}^{r}(\omega) \\
\mathcal{F}_{j}^{r}(\omega)+\mathbf{F}_{\Sigma, j}^{r}(\omega)
\end{array}\right] .
\end{aligned}
$$

with $\mathcal{F}_{i}^{r}(\omega)=\left[P^{r}\right]^{T} \mathbf{F}_{i}^{r}(\omega)$ and $\mathcal{F}_{j}^{r}(\omega)=$ $\left[\Phi_{i j}^{r}\right]^{T} \mathbf{F}_{i}^{r}(\omega)+\mathbf{F}_{j}^{r}$, and we have

$$
\left[\mathcal{A}^{r}(\omega)\right]\left[\begin{array}{c}
\mathbf{q}^{r}(\omega) \\
\mathbf{U}_{j}^{r}(\omega)
\end{array}\right]=\mathcal{F}^{r}(\omega)
$$

\subsection{Reduced matrix model for structure}

$$
\Omega=\Omega_{1} \cup \Omega_{2} \text {. }
$$

Using the coupling conditions on interface $\Sigma$ defined by Eqs. (8)-(9) and the reduced matrix models for substructures $\Omega_{1}$ and $\Omega_{2}$ defined by Eqs. (16)-(18), yields

$$
[\mathcal{A}(\omega)]\left[\begin{array}{c}
\mathbf{q}^{1}(\omega) \\
\mathbf{q}^{2}(\omega) \\
\mathbf{U}_{j}(\omega)
\end{array}\right]=\mathcal{F}(\omega)
$$

in which

$$
\begin{gathered}
{[\mathcal{F}(\omega)]=\left[\begin{array}{c}
\mathcal{F}_{i}^{1}(\omega) \\
\mathcal{F}_{i}^{2}(\omega) \\
\mathcal{F}_{j}^{1}(\omega)+\mathcal{F}_{j}^{2}(\omega)
\end{array}\right],} \\
{[\mathcal{A}(\omega)]=} \\
{\left[\begin{array}{lll}
{\left[\mathcal{A}_{i i}^{1}(\omega)\right]} & {[0]} & {\left[\mathcal{A}_{i j}^{1}(\omega)\right]} \\
{[0]} & {\left[\mathcal{A}_{i i}^{2}(\omega)\right]} & {\left[\mathcal{A}_{i j}^{2}(\omega)\right]} \\
{\left[\mathcal{A}_{i j}^{1}(\omega)\right]^{T}} & {\left[\mathcal{A}_{i j}^{2}(\omega)\right]^{T}} & {\left[\mathcal{A}_{j j}(\omega)\right]}
\end{array}\right],}
\end{gathered}
$$

in which $\left[\mathcal{A}_{j j}(\omega)\right]=\left[\mathcal{A}_{j j}^{1}(\omega)\right]+\left[\mathcal{A}_{j j}^{2}(\omega)\right]$. 


\section{Construction of $\left[P^{r}\right]$ for the re- duced matrix model of a sub- structure with fixed coupling interface in the MF range.}

\subsection{Finite element discretization of the spectral problem related to the me- chanical energy operator.}

For the construction of $\left[P^{r}\right]$, we consider the particular external forces relative to substructure $\Omega_{r}$ with fixed coupling interface $\Sigma$, defined by $\mathbf{F}_{i}^{r}(\omega)=$ $\eta(\omega) \mathbf{F}_{0}^{r}$ in which $\mathbf{F}_{0}^{r}$ is a $\mathbb{C}^{n_{r}-m}$-valued vector independant of $\omega$ and where $\omega \rightarrow \eta(\omega)$ is a complexvalued function defined on $\mathbb{R}$ such that $\eta(\omega)=0$ if $\omega \notin B \cup \widetilde{B}$ and such that $|\eta(\omega)|=|\eta(-\omega)|$. Nodal displacement vector $\mathbf{U}_{i}^{r}(\omega)$ of substructure $\Omega_{r}$ with fixed coupling interface $\Sigma$ is such that

$$
\left[A_{i i}^{r}(\omega)\right] \mathbf{U}_{i}^{r}(\omega)=\eta(\omega) \mathbf{F}_{0}^{r} .
$$

For all $\Omega$ in $B \cup \widetilde{B}$, matrix $\left[A_{i i}^{r}(\omega)\right]$ is invertible and $\left[T_{i i}^{r}(\omega)\right]=\left[A_{i i}^{r}(\omega)\right]^{-1}$ is the matrix-valued frequency response function. The finite element approximation of the eigenvalue problem related to the mechanical energy operator of substructure $\Omega_{r}$ with fixed coupling interface $\Sigma$, relative to MF band $B \cup \widetilde{B}$, is written (see [8]-[9]) as

$$
\left[G^{r}\right]\left[E_{B}^{r}\right]\left[G^{r}\right] \mathbf{P}^{r}=\lambda^{r}\left[G^{r}\right] \mathbf{P}^{r},
$$

in which $\mathbf{P}^{r} \in \mathbb{R}^{n_{r}-m}$ is the eigenvector associated with the positive real eigenvalue $\lambda^{r},\left[G^{r}\right]$ is the positive-definite symmetric $\left(n_{r}-m, n_{r}-m\right)$ real matrix corresponding to the finite element discretization of the bilinear form $(u, v) \rightarrow \int_{\Omega_{r}} \mathbf{u}(\mathbf{x}) \cdot \mathbf{v}(\mathbf{x}) d \mathbf{x}$ and where $\left[E_{B}^{r}\right]$ is the positive-definite symetric $\left(n_{r}-\right.$ $\left.m, n_{r}-m\right)$ real matrix which is written as

$$
\left[E_{B}^{r}\right]=\frac{1}{\pi} \int_{B} \omega^{2}|\eta(\omega)|^{2}\left[e_{n}^{r}(\omega)\right] d \omega \quad,
$$

in which

$$
\left[e_{n}^{r}(\omega)\right]=\Re \mathfrak{e}\left\{\left[T_{i i}^{r}(\omega)\right]^{*}\left[M_{i i}^{r}\right]\left[T_{i i}^{r}(\omega)\right]\right\},
$$

where $\Re \mathfrak{e}$ is the real part of complex number and where $\left[T_{i i}^{r}(\omega)\right]^{*}={\overline{\left[T_{i i}^{r}(\omega)\right]}}^{T}$ is the adjoint matrix. It should be noted that $\left[E_{B}^{r}\right]$ depends on MF band $B$, but does not depend on the spatial part of the external excitation represented by $\mathbf{F}_{0}^{r}$. The columns of $\left(n_{r}-m, N_{r}\right)$ real matrix $\left[P^{r}\right]$ introduced in section 2.1 are the eigenvectors $\mathbf{P}_{1}^{r}, \ldots, \mathbf{P}_{N_{r}}^{r}$ associated with the $N_{r}$ highest eigenvalues $\lambda_{1}^{r} \geq \lambda_{2}^{r} \geq \ldots \geq \lambda_{N_{r}}^{r}$ of the generalized eigenvalue problem defined by Eq. (23). We then have

$$
\left[P^{r}\right]=\left[\begin{array}{lll}
\mathbf{P}_{1}^{r} & \ldots & \mathbf{P}_{N_{r}}^{r}
\end{array}\right]
$$

\subsection{Energy method implementation.}

For each substructure $\Omega_{r}$ with fixed coupling interface $\Sigma$, we have to compute the $N_{r}$ highest eigenvalues and corresponding eigenvectors of the generalized symmetric eigenvalue problem with positive-definite matrices defined by Eq. (23) that we can rewrite as

$$
\begin{aligned}
{\left[G^{r}\right]\left[E_{B}^{r}\right]\left[G^{r}\right]\left[P^{r}\right] } & =\left[G^{r}\right]\left[P^{r}\right]\left[\Lambda^{r}\right], \\
{\left[P^{r}\right]^{T}\left[G^{r}\right]\left[P^{r}\right] } & =\left[I_{n_{r}-m}\right],
\end{aligned}
$$

in which Eq. (28) defines the normalization and where $\left[\Lambda^{r}\right]$ is the diagonal matrix of the eigenvalues $\lambda_{1}^{r}, \lambda_{2}^{r}, \ldots, \lambda_{N_{r}}^{r}$.

Consequently, using the subspace iteration method (see for instance [1]) and introducing matrix $[R]$ such that $\left[R^{r}\right]=\left[G^{r}\right]\left[E_{B}^{r}\right]\left[G^{r}\right]$, we have to calculate the lowest eigenvalues and corresponding eigenvectors of the following generalized eigenvalue problem

$$
\begin{aligned}
& {\left[G^{r}\right]\left[S^{r}\right]=\left[R^{r}\right]\left[S^{r}\right]\left[\Gamma^{r}\right],} \\
& {\left[S^{r}\right]^{T}\left[R^{r}\right]\left[S^{r}\right]=\left[I_{n_{r}-m}\right],}
\end{aligned}
$$

in which $\left[\Gamma^{r}\right]=[\Lambda]^{-1}$ and $\left[P^{r}\right]=\left[S^{r}\right]\left[\Gamma^{r}\right]^{-1 / 2}$. The dimension of the subspace used in the subspace iteration method is equal to $\nu=\min \left\{2 N_{r}, N_{r}+\right.$ $8\}$. To solve the eigenvalue problem defined by Eqs. (29)-(30), matrix $\left[E_{B}^{r}\right]$ is not explicitly calculed. An indirect procedure is used (see Ref. [8]). For each iteration of the subspace iteration algorithm, we only need to calculate a $\left(n^{r}-m, \nu\right)$ real matrix $\left[W^{r}\right]=\left[E_{B}^{r}\right]\left[X^{r}\right]$, in which $\left[X^{r}\right]$ is a given $\left(n^{r}-m, \nu\right)$ real matrix. For $\omega$ in $B$, the approximations $\left[D^{r}(\omega)\right] \simeq\left[D^{r}\left(\omega_{B}\right)\right],\left[K^{r}(\omega)\right] \simeq\left[K^{r}\left(\omega_{B}\right)\right]$ are used and it is proved that $\left[W^{r}\right]$ can be calculated by $\left[W^{r}\right]=2 \pi \Re \mathfrak{e}\left[Z_{0}^{r}(0)\right]$ in which $\left[Z_{0}^{r}(t)\right]$ is the solution of LF equations in time domain associated with MF equations, these LF equations being written in the time domain as

$$
\begin{array}{r}
{\left[M^{r}\right]\left[\ddot{Y}_{0}^{r}(t)\right]+\left[\widetilde{D}_{B}^{r}\right]\left[\dot{Y}_{0}^{r}(t)\right]+\left[\widetilde{K}_{B}^{r}\right]\left[Y_{0}^{r}(t)\right]} \\
=\Psi_{0}(t)\left[X^{r}\right], \\
{\left[M^{r}\right]\left[\ddot{Z}_{0}^{r}(t)\right]+\left[\widetilde{D}_{B}^{r}\right]\left[\dot{Z}_{0}^{r}(t)\right]+\left[\widetilde{K}_{B}^{r}\right]\left[Z_{0}^{r}(t)\right]} \\
=\left[M^{r}\right]\left[Y_{0}^{r}(-t)\right],
\end{array}
$$


in which $\Psi_{0}(t)$ is the inverse Fourier transform of $\widehat{\Psi}_{0}(\omega)=\widehat{\Psi}\left(\omega+\omega_{B}\right)$ with $\widehat{\Psi}(\omega)=$ $\frac{1}{\pi} \omega^{2}|\eta(\omega)|^{2} \mathbf{1}_{B}(\omega)$, and

$\left[\widetilde{K}_{B}^{r}\right]=-\omega_{B}^{2}\left[M^{r}\right]+i \omega_{B}\left[D^{r}\left(\omega_{B}\right)\right]+\left[K^{r}\left(\omega_{B}\right)\right]$,

$\left[\widetilde{D}_{B}^{r}\right]=\left[D^{r}\left(\omega_{B}\right)\right]+2 i \omega_{B}\left[M^{r}\right]$

The LF equations (31) and (32) associated with the MF frequency band $B$, are solved by using the Newmark method ([1]). The sampling time step is given by Shannon's theorem $\tau=2 \pi / \Delta \omega$ and the integration time step of the step-by-step integration method is written as $\Delta t=\tau / \mu$ in which $\mu>1$ is an integer. the initial time $t_{I}$ and the final time $t_{F}$ are respectively defined by $t_{I}=-I_{0}$ and $t_{F}=J_{0}$ in which $I_{0}>1$ and $J_{0}>1$ are integers. The details of the method can be found in [8].

\section{Example}

We consider a rectangular, homogeneous, isotropic thin plate, simply supported, with a constant thickness $0.4 \times 10^{-3} \mathrm{~m}$, width $0.5 \mathrm{~m}$, length $1.0 \mathrm{~m}$, mass density $7800 \mathrm{~kg} / \mathrm{m}^{3}$, constant damping rate 0.01, Young's modulus $2.1 \times 10^{11} \mathrm{~N} / \mathrm{m}^{2}$, Poisson's ratio $\nu=0.29$. Two point masses of $3 \mathrm{~kg}$ and $4 \mathrm{~kg}$ are located at points $(0.2,0.4)$ and $(0.35,0.75)$, and three springs having the same stiffness coefficient $2.388 \mathrm{~N} / \mathrm{m}$ are attached normally to the plate and located at points $(0.22,0.28),(0.33,0.54)$ and $(0.44,0.83)$. Consequently, the master structure defined above is not homogeneous. This master structure is decomposed into two substructures (see Figure 4). The first one has a length $0.6 \mathrm{~m}$ and the second one, a length $0.4 \mathrm{~m}$

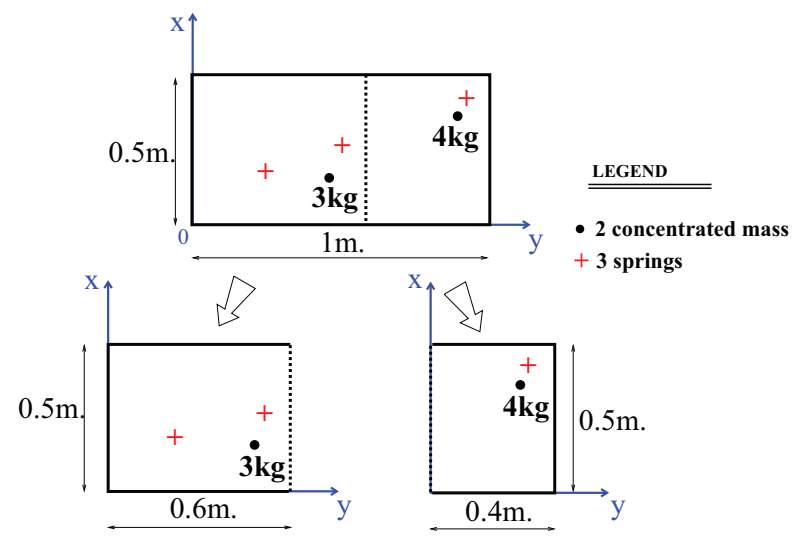

Figure 4: Master structure (a simply supported plate in bending mode) decomposed into two substructures.
We are interested in the prediction of the response of the coupled substructures in the MF narrow band $B_{1}=2 \pi \times[500,550] \mathrm{rad} / \mathrm{s}$ and the MF broad band $B_{2}=2 \pi \times[450,650] \mathrm{rad} / \mathrm{s}$. The validation of dynamic substructuring method in the MF range presented above is obtained in comparing the frequency response functions calculed for the coupled substructures with those which are directly calculed for the master structure. The finite element model is constructed using 4-nodes bending plate elements. The finite element mesh of the master structure is shown in Figure 5. The mesh size is $0.01 \mathrm{~m} \times 0.01 \mathrm{~m}$ and numerical informations are summarized in tables 1 and 2 . We have $m=149, n_{1}=8989$ and $n_{2}=6009$. The total number of DOFs of the master structure is $n=\left(n_{1}-m\right)+\left(n_{2}-m\right)+m=14849$.

\begin{tabular}{|c|c|c|c|}
\hline & $\begin{array}{c}\text { Number } \\
\text { of nodes }\end{array}$ & $\begin{array}{c}\text { Number } \\
\text { of DOFs }\end{array}$ & Matrix size \\
\hline Master plate & 5151 & 14849 & $14849 \times 14849$ \\
\hline Subplate 1 & 3111 & 8989 & $8989 \times 8989$ \\
\hline Subplate 2 & 2091 & 6009 & $6009 \times 6009$ \\
\hline
\end{tabular}

Table 1 : Number of nodes and DOFs, size of matrices.

\begin{tabular}{|c|c|c|c|}
\hline & Master plate & Subplate 1 & Subplate 2 \\
\hline Stiffness matrix & 348997 & 211103 & 140639 \\
\hline Mass matrix & 42775 & 25810 & 17110 \\
\hline
\end{tabular}

Table 2 : Number of nonzeros entries in the matrices of the finite element model.

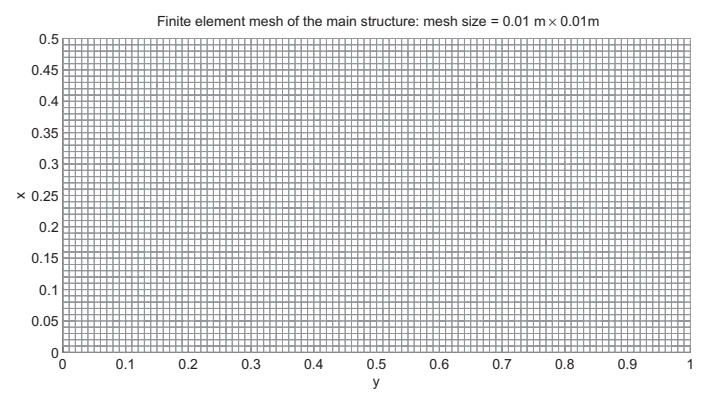

Figure 5: Finite element mesh of the master structure.

For each structure, it is assumed that damping matrix is proportional to its stiffness matrix with a damping coefficient $\theta=2 \xi / \omega_{B_{1}}$ with $\xi=0.01$. The master structure is submitted to a random excitation $\{\mathbf{F}(t), t \in \mathbb{R}\}$ which is an $\mathbb{R}^{n}$-valued mean-square stationary centered second-order stochastic process indexed by $\mathbb{R}$ whose matrix-valued spectral density function $\left[S_{\mathbf{F}}(\omega)\right]$ is written as $\left[S_{\mathbf{F}}(\omega)\right]=[B][B]^{T}$. 
The entries of $(n \times s)$ real matrix [B], with $s=50$, are 0 or 1 and matrix $[B]$ is such that $[B]^{T}[B]=$ $\left[I_{s}\right]$. The DOFs excited correspond to the normal displacements at nodes uniformly distributed over the master plate. The mean-square stationary response $\{\mathbf{U}(t), t \in \mathbb{R}\}$ of the master structure is $\mathbb{R}^{n}$ valued second-order stochastic process whose matrixvalued spectral density function $\left[S_{\mathbf{U}}(\omega)\right]$ is written as $\left[S_{\mathbf{U}}(\omega)\right]=[T(\omega)]\left[S_{\mathbf{F}}(\omega)\right][T(\omega)]^{*}$ in which $[T(\omega)]$ is the matrix-valued frequency response function of the master structure. We then deduce that $\left[S_{\mathbf{U}}(\omega)\right]=[\mathbb{U}(\omega)][\mathbb{U}(\omega)]^{*}$ with $\mathbb{U}=[T(\omega)][B]$. We then introduce the power spectral density function $e(\omega)=\operatorname{tr}\left\{\left[S_{\mathbf{U}}(\omega)\right]\right\}$ which can be written as $e(\omega)=\operatorname{tr}\left\{[\cup(\omega)][\cup(\omega)]^{*}\right\}$.

Figure 6 shows the graph of function $\nu \mapsto$ $10 \log _{10} e(2 \pi \nu)$ for the master structure on the $[0,650] \mathrm{Hz}$ broad frequency band. This graph defines the reference solution

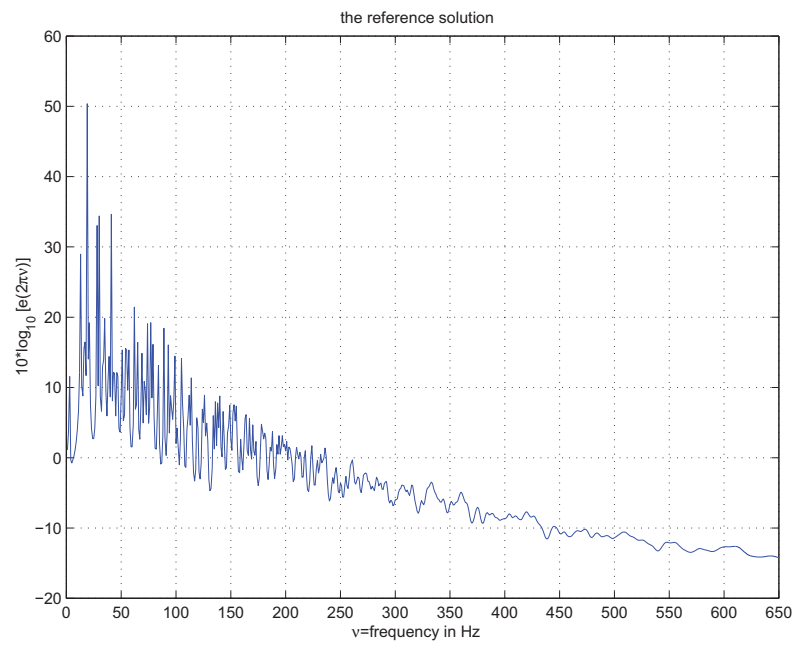

Figure 6: Convergence of reduced model over the $[500,550] \mathrm{Hz}$ narrow band for the master structure.

Figures 7 to 9 are correspond to results obtained by MF dynamic substructuring. For frequency band $B_{1}$ and for each substructure $\Omega_{r}$ with fixed coupling interface, the distribution of highest eigenvalues $X_{1} \geq$ $\lambda_{2}^{r} \geq \ldots$ of the generalized eigenvalue problem defined by Eq. (23) is shown in Figure 7. For each substructure, there is a strong decrease in the eigenvalues which means there exists possibility of constructing an efficient reduced model for this substructure in the MF range. For frequency band $B_{1}$, order $N_{r}$ of the reduced model is 40 for $r=1$ and 30 for $r=2$. In figure 8 , each dashed line corrresponds to the response $\nu \mapsto 10 \log _{10} e(2 \pi \nu)$ calculated with the MF dynamic substructuring method for different values of $N_{1}$ and $N_{2}$. The solid line corresponds to the reference solution; this figure shows the convergence of the MF dynamic substructuring method as $N_{1}$ and $N_{2}$ increase.
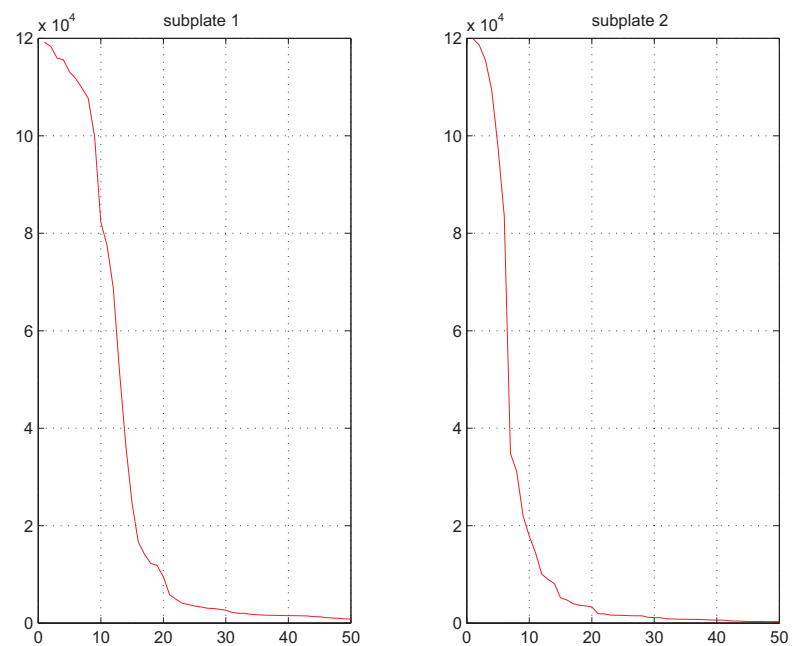

Figure 7: Graph of function $k \mapsto \lambda_{k}^{r}$ for $k=1, \ldots, 50$, showing the distribution of eigenvalues of energy operator for $r=1$ (graph on the the left) and $r=2$ (graph on the the right).

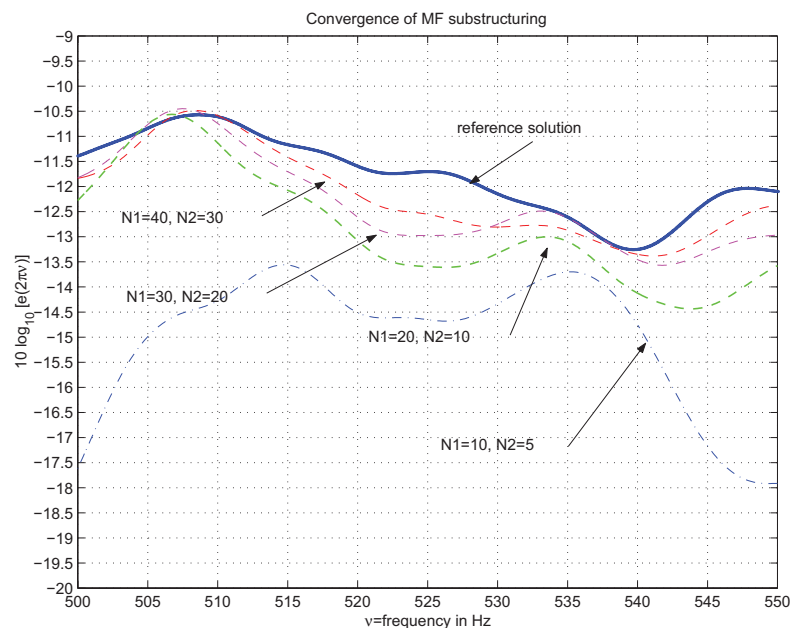

Figure 8: MF dynamic substructuring results for the $[500,550] \mathrm{Hz}$ narrow frequency band: convergence with respect to $N_{1}$ and $N_{2}$

Finally, Figure 9 shows the comparison between the reference solution and the MF dynamic substructuring solution calculed with $N_{1}=40$ and $N_{2}=30$ for broad frequency band $B_{2}$ which is written as union of four narrow frequency bands. 


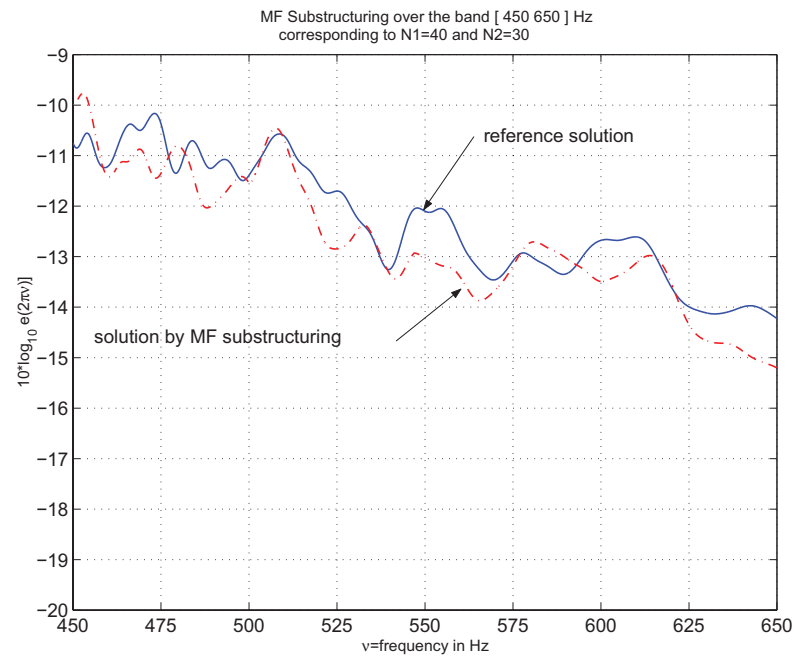

Figure 9: MF dynamic substructuring results for the [450, 650] broad frequency band corresponding to $N_{1}=40$ and $N_{2}=30$.

\section{Conclusion}

The numerical results obtained correspond to a first validation of the dynamic substructuring method in the medium-frequency range presented in this paper. These first results are good enough and more advanced validations are in progress.

\section{References}

1. K.J. Bathe and E.L. Wilson, Numerical Methods in Finite Element Analysis (Prentice Hall, New York, 1976).

2. R.R. Craig and M.C.C. Bampton, Coupling of Substructures for Dynamic Analysis, AIAA Journal, 6(7), pp. 1313-1319 (1968).

3. W.C. Hurty, Dynamic Analysis of Structural Systems Using Component Modes, AIAA Journal, 3(4), pp. 678-685 (1965).

4. H. J. P. Morand and R. Ohayon, Substructure Variational Analysis for the Vibrations of Coupled Fluid-Structure Systems, Int. Num. Meth. Eng., 14(5), pp. 741-755 (1979).

5. H. J. P. Morand and R. Ohayon, Fluid Structure Interaction (John Wiley and Sons, New York, 1995).

6. R. Ohayon, R. Sampaio, C. Soize, Dynamic Substructuring of Damped Structures Using Singular Value Decomposition, Journal of Applied Mechanics, 64, pp. 292-298 (1997).
7. R. Ohayon and C. Soize, Structural Acoustics and Vibration (Academic Press, San Diego, London, 1998).

8. C. Soize, Reduced Models in the Medium Frequency Range for General Dissipative Structural-Dynamics Systems, European Journal of Mechanics, A/Solid, 17(4), pp. 657-685 (1998).

9. C. Soize, Reduced Models in the MediumFrequency Range for General External Structural-Acoustic Systems, J. Acoust. Soc. Am., 103 (6), pp. 3393-3406 (1998).

10. C. Soize, Medium Frequency Linear Vibrations of Anisotropic Elastic Structures, La recherche aerospatial (English edition), 5, pp. 65-87 (1982). 